\title{
Can Polycystic Ovarian Syndrome be cured? Unfolding the Concept of Secondary Polycystic Ovarian Syndrome!
}

\author{
Suvarna Satish Khadilkar ${ }^{1}$
}

Received: 5 July 2019 / Accepted: 5 July 2019 / Published online: 18 July 2019

(c) Federation of Obstetric \& Gynecological Societies of India 2019

\begin{abstract}
Classic polycystic ovarian syndrome (PCOS) is one of the commonest endocrine metabolic disorders in women of reproductive years. It is very disappointing that we have still not been able to know the exact cause, and hence, we cannot completely cure this syndrome. With availability of effective drugs and lifestyle modification, it can only be controlled and symptoms can be reduced. There are some well-defined factors which lead to PCOS phenotype. A concept of secondary PCOS is being described recently. This condition, even though rare, is completely curable, as majority of these factors are treatable. Hence, it is advisable to actively look for these conditions and offer treatment in time. This editorial gives an account of various causes of secondary PCOS, gives broader view of PCOS phenotypes and aims for an insight into pathophysiological aspects of PCOS. The clinical categorization by HA-PODS nomenclature of both primary and secondary PCOS can minimize diagnostic and therapeutic pitfalls and will serve as a checklist to ensure the appropriate investigation is ordered and specific treatment is initiated as per diagnostic code. This will achieve uniformity in therapies offered and also enable better comparison of data and a smooth conduct of research which is much needed in this field.
\end{abstract}

Keywords Polycystic ovarian syndrome · HA-PODS · Secondary PCOS · Insulin resistance $\cdot$ Hyperprolactinaemia $\cdot$ Hyperandrogenaemia $\cdot$ Menstrual dysfunction $\cdot$ Hypothyroidism $\cdot$ Autoimmune thyroiditis $\cdot$ Congenital adrenal hyperplasia $\cdot$ Obesity

\section{Introduction}

Polycystic ovarian syndrome (PCOS) is a heterogeneous disorder with multiple phenotypes. Despite the fact that the condition was identified in 1935 [1], we have not succeeded in finding a cure! The reason is that we have still not zeroed down on the exact aetiology of this syndrome. We know all the possible symptoms it can produce, and we have

Prof. Suvarna Satish Khadilkar MD DGO FICOG, CIMP, Diploma in Endocrinology (UK), is Editor in Chief of Journal of Obstetrics and Gynecology of India, and Treasurer, FOGSI; she is Professor and Head, and Consultant Gyne-Endocrinologist, Bombay Hospital and Medical Research Centre, Mumbai, Former Professor and Head, Dept of Ob-Gyn, RCSM, Government Medical College, Maharashtra, and Associate Professor and Unit Chief Grant Medical College and Cama and Albless Hospital, Mumbai.

Prof. Suvarna Satish Khadilkar suvarnakhadilkar2@gmail.com

1 Bombay Hospital and Medical Research Centre, Mumbai, India more recently understood that it has a lot of metabolic consequences, but disappointingly not understood the precise cause. Hence, we can only reduce or eliminate symptoms but not the pathology.

We have subsequently discovered that some hyperandrogenic conditions result in polycystic ovarian syndrome phenotype. The concept of secondary PCOS has emerged due to this fact that there are some well-defined conditions which induce PCOS-like syndrome. So clinicians can identify these causes and completely cure the consequent PCOS as most of them are treatable conditions.

Various diagnostic criteria have been proposed by different groups like NIH, Rotterdam group, and androgen excess and PCOS society for classic PCOS [2-5], and all of them have one common prerequisite, exclusion of the mimics. However, the core diagnostic features of PCOS [hyperandrogenism, persistent ovulatory dysfunction and polycystic ovarian morphology (PCOM)] have been variable in these diagnostic criteria. To prevent diagnostic and therapeutic pitfalls, a new nomenclature "HA-PODS (hyperandrogenism and persistent ovarian dysfunction syndrome)" was proposed 
which reflects exact clinico-pathological aspects [6]. After several years of experience, I am of the view that longstanding ovulatory dysfunction and long-standing hyperandrogenism perpetuate each other irrespective of the cause. This vicious cycle of one precipitating the other (Fig. 1) will eventually produce PCOS phenotype irrespective of the initiating mechanism. We have clearer understanding today that primary defects in HPO axis will lead to the classic PCOS, and factors other than the HPO axis will lead to the secondary PCOS. In my personal case series of 100 PCOS cases, I observed hyperprolactinaemia in $12 \%$ and thyroid disorders in $18 \%$ as the cause of persistent anovulation, and after treatment, many of them resolved and started getting regular cycles (unpublished data).

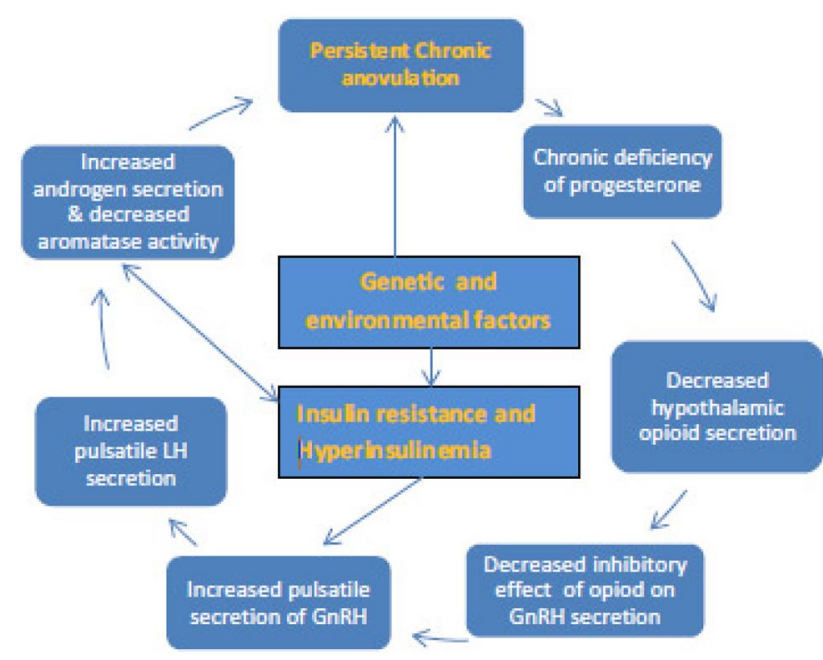

Fig. 1 Vicious cycle of persistent anovulation. Reproduced with permission [6]

\section{Aetiological Classification of PCOS}

Primary PCOS: Primary PCOS is the most common variety of PCOS without any known cause. Proposed theory suggests that there is functional ovarian hyperandrogenism (FOH) along with disturbance of hypothalamo-pituitary-ovarian axis function.

Secondary PCOS: The PCOS arising due to well-defined causes other than HPO axis dysfunction is labelled as secondary PCOS.

Causes of secondary PCOS are listed in Table 1.

\section{Clinical Categories as Per Core Features and Comorbidities}

As per AE-PCOS society definition [5] at least one from each group must be present: clinical or biochemical evidence of hyperandrogenism and clinical, biochemical or sonographic evidence (PCOM) of persistent ovulatory dysfunction.

Author described new nomenclature, "HA-PODS""hyperandrogenic persistent ovulatory dysfunction syndrome" [6]. With unfolding the concept the secondary PCOS, the nomenclature has added secondary PCOS categories (Table 2).

In my practice, this nomenclature is extremely useful to prevent diagnostic pitfalls and to plan the management. All patients get a diagnostic code at the end of complete investigations, depending on which of the above abnormalities are detected. For example, if there are insulin resistance and hypertension, the patient's diagnostic code is HA-PODSIR-HT; if patient also has endometrial hyperplasia, then the diagnostic code is written as HA-PODS-IR-HT-EH. If

Table 1 Secondary causes mimicking PCOS

\begin{tabular}{|c|c|c|c|c|}
\hline \multirow{2}{*}{$\begin{array}{l}\text { Adipose tissue and Skin } \\
\text { Obesity }\end{array}$} & \multirow{2}{*}{$\begin{array}{l}\text { Thyroid } \\
\text { Hypothyroidism }\end{array}$} & Pituitary & \multicolumn{2}{|c|}{ Adrenal } \\
\hline & & Prolactinoma & $\begin{array}{l}\text { Glucocorticoid-suppressible } \\
\text { FAH }\end{array}$ & $\begin{array}{l}\text { Glucocorticoid-non-suppressible } \\
\text { FAH }\end{array}$ \\
\hline Lipodystrophy syndromes & Autoimmune thyroiditis & Hyperprolactinaemia & $\begin{array}{l}\text { CAH: classic and non-classic } \\
\text { 21-hydroxylase deficiency }\end{array}$ & Cushing's syndrome \\
\hline SSIR & & $\begin{array}{l}\text { ACTH secreting } \\
\text { tumour, Cushing's } \\
\text { disease }\end{array}$ & $\begin{array}{l}\text { Other less common enzymes } \\
\text { deficiency }^{\mathrm{a}}\end{array}$ & Androgen-secreting tumours \\
\hline Idiopathic Hirsuitism & & \multicolumn{2}{|l|}{ Acromegaly } & Glucocorticoid resistance \\
\hline Ovaries & \multicolumn{3}{|c|}{ Neuroendocrine } & Drug induced \\
\hline DSD & \multicolumn{3}{|c|}{ Epilepsy } & Androgenic drugs \\
\hline \multicolumn{4}{|c|}{ Androgen-secreting tumours \{sertoli leydig cell tumour $\}$} & Valproic acid \\
\hline
\end{tabular}

$F A H$ functional adrenal hyperandrogenism, DSD disorders of sex development, SSIR syndrome of severe insulin resistance, $C A H$ congenital adrenal hyperplasia

${ }^{a} 11$ Beta-hydroxylase, cortisone reductase deficiency, DHEA sulphotransferase deficiency 
Table 2 Clinical and aetiological categories of PCOS

\begin{tabular}{llll}
\hline HA-PODS-primary & HA-PODS-secondary \\
\hline IR & Insulin resistance & PRL & Hyperprolactinaemia \\
O & Obesity & THY & Thyroid disorders \\
DM & Diabetes mellitus & CAH & Congenital adrenal hyperplasia \\
D & Dyslipidaemia & SSIR & Syndrome of severe insulin resistance \\
C-EH & Cancer/endometrial hyperplasia & LD & Lipodystrophy \\
HT & Hypertension & AST & Androgen-secreting tumour \\
SA & Sleep apnoea & Cush & Cushing's syndrome \\
CV & Cardiovascular disease & DI & Drug (Valproic acid) Induced \\
FL & Fatty liver & & \\
MS & Metabolic syndrome & & \\
\hline
\end{tabular}

secondary cause is detected, diagnostic code specifies the same along with causative factor; for example, if there is thyroid disorder, then it will be labelled as secondary HAPODS-THY. If any co-morbidity is also present then the respective diagnostic code can be added and then the codespecific treatment can be started for each factor. HA-PODS or PCOS phenotype gets completely resolved in case of treatable causes.

\section{Common Conditions Mimicking PCOS}

\section{Obesity}

Whether obesity is the cause, effect or incidental comorbidity is not very clear. It has been reported that if significant weight loss happens in women with extreme obesity, the PCOS-like picture undergoes complete remission in large number of women. And likewise excessive weight gain at or after puberty produces PCOS-like condition. The weight loss can occur because of diet, exercise and lifestyle changes, or severe weight loss can occur due to bariatric surgery. Whatever is the cause of weight loss, many women undergo complete remission as long as weight is under control [7].

One study reported $36.9 \%$ complete recovery from all features of PCOS with weight reduction, and only $15.4 \%$ had persistent PCOS features [8].

Impact of bariatric surgery on weight loss is well documented [9]. A recent meta-analysis showed that preoperative incidence of PCOS (45.6\%) significantly decreased to $6.8 \%$ $(p<0.001)$, menstrual irregularities, hirsutism also reduced significantly, and many of them achieved complete remission at 12-month follow-up after bariatric surgery [10].

So obesity could be an independent secondary causal association of PCOS.

\section{Thyroid Disorders and PCOS}

Hypothyroidism and Hashimoto thyroiditis are commonly found associated in women with PCOS. It is certainly more prevalent than in general population. General gynaecologist gets confused whether such patients are to be diagnosed as PCOS or no because this is contrary to recommendation that we must rule out thyroid disorders before diagnosing PCOS. The most logical explanation is that thyroid disorders particularly hypothyroidism often leads to persistent anovulation or ovulatory dysfunction. Longer the duration of the dysfunction, more sustained is the impact of anovulation. The resultant lack of progesterone triggers the vicious cycle of PCOS (Fig. 1), thereby leading to hyperandrogenism. But this does not happen in all women with thyroid disorders. It happens more commonly in genetically susceptible individuals. Common genetic predisposition exists for the development of both the diseases. The three genetic polymorphisms such as FBN3, GnRHR and CYP1B1 play a role in both the diseases [11].

Apart from genetic susceptibility, there are other common pathways which lead to both the diseases. The role of transforming growth factor beta (TGF- $\beta$ ) a multifunctional cytokine is implicated in the pathogenesis of both disorders. High levels of TGF- $\beta$ are more prone to develop autoimmune diseases.

High oestrogen levels during intrauterine life interfere with the thymus development hence could cause both hypothyroidism and PCOS. Women with PCOS have imbalance between various sex steroids.

Unopposed oestrogen in the presence of high androgens stimulates autoimmunity. Hence, PCOS could lead to autoimmune thyroiditis. To sum up long-standing thyroid disorders leads to ovulatory dysfunction and lead to PCOS, whereas PCOS could lead to autoimmune thyroid disorder. Vitamin D deficiency is also proposed to be the common predisposing factor for both the disorders [12].

Polycystic appearance of ovaries sometimes referred to as soap bubble appearance on ultrasound is common with frank hypothyroidism, and milder forms may mimic polycystic morphology. We have effective therapy for thyroid disorders; hence, the progression of the disease could be halted with LT4. Vitamin D deficiency can also 
be treated effectively today. Remission therefore is likely in this coexistence [13].

\section{Hyperprolactinaemia}

Elevated prolactin levels are found in $6 \%$ of women with hirsutism [14]. The exact reason why elevated prolactin levels are associated with androgen excess is not very clear. Direct action of prolactin on adrenal cortex is hypothesized. In my view, hyperprolactinaemia leads to chronic persistent ovulatory dysfunction with sustained lack of progesterone which may trigger the vicious cycle of PCOS and hyperandrogenism (Fig. 1). Fortunately, we have very effective treatment of this condition like cabergoline and dopamine agonists. The reversal of PCOS phenotype after treatment is quite common [14].

\section{Adrenal Hyperandrogenic Disorders}

\section{a. Congenital Adrenal Hyperplasia}

Congenital adrenal hyperplasia $(\mathrm{CAH})$ is a group of disorders arising due to deficiency of enzymes of steroidogenesis. All forms of CAH, including 21-hydroxylase deficiency, are transmitted as autosomal recessive disorders. Women carrying a classic mutation will transmit this to next generation which will be affected with severe disease. Conversion of 17-hydroxyprogesterone to 11-deoxycortisol and cortisol is blocked (CYP21 A2); hence, there is hypocortisolism. Cortisol has a negative feedback on ACTH secretion, so hypocortisolism results in excessive secretion of ACTH which brings about hyperplasia and hyperactivity of all the three zones of adrenal gland, and high levels of 17-hydroxyprogesterone levels and androstenedione.

Female newborns when referred for ambiguous genitalia, with more serious form of salt wasting type and less risky virilising forms of $\mathrm{CAH}$, are examples of classical 21-hydroxylase deficiency, but this form is mostly diagnosed in infancy. The "non-classical" form of 21-hydroxylase deficiency does not become apparent until adolescence or early adulthood, when abnormally high androgen levels cause hirsutism, menstrual abnormalities, premature pubarche and a phenotype that mimics polycystic ovary syndrome (PCOS). Prevalence of non-classic 21-hydroxylase deficiency ranges from 1 to $3 \%$ in general [15] but may be higher in tertiary referral centres.

Severe forms may require prednisolone treatment, but non-classic variety generally does not require any treatment till they become symptomatic. They will require ovulation induction and antiandrogen therapy to control the symptoms. Follicular phase 17-hydroxyprogesterone estimation must be done in all hyperandrogenic adolescents to rule out CAH. ACTH stimulation test may be done though not mandatory to confirm diagnosis of CAH. This well-defined secondary cause of PCOS is non-curable as it is a congenital disorder, but this can certainly be effectively controlled and symptoms may be relieved.

\section{b. Cushing's Syndrome}

Cushing's syndrome is defined as hypercortisolism due to primary adrenal pathology often associated with obesity, hypertension and type II diabetes. If this results from primary pathology in pituitary gland secreting excess ACTH, it is termed as Cushing's disease. It gives rise to PCOS phenotype. In fact many a times, they are simply treated as PCOS cases which are resistant to standard treatment. PCOS is very common presentation, but Cushing's syndrome is relatively rare. However, we must remember that if diagnosed early, Cushing's syndrome will be completely cured unlike PCOS. So suspecting diagnosis is very important. Suspicion should arise when clinical features like plethoric round faces, supraclavicular fat pads and abdominal purple striae are found in addition to classic features of PCOS, i.e. central obesity, hirsutism, acne, baldness and irregular menstruation. These clinical indicators justify diagnostic tests like low-dose dexamethasone suppression test, $24 \mathrm{~h}$ urinary free cortisol and midnight salivary cortisol estimation, to diagnose Cushing's syndrome. One survey reported that only $6 \%$ of gynaecologists screened PCOS referrals for Cushing's syndrome [16]. Treatment in the form prednisolone medication, or surgery like laparoscopic adrenalectomy or transsphenoidal resection of ACTH secreting pituitary tumour may lead to complete cure of this condition.

\section{Syndrome of Severe Insulin Resistance Syndrome (SSIR)}

Severe insulin resistance syndrome (SSIR) is a group of disorders based on severe insulin resistance irrespective of whether patient is obese or non-obese. SSIR can be subclassified by the type of defect that leads to the severe insulin resistance. Type of defects includes autoantibodies, mutations and defects in signalling pathways of insulin receptors. A defect in the adipose tissue function leads to different types of lipodystrophies $[17,18]$. There are several lipodystrophic phenotypes. These occur because of loss of subcutaneous fat which can be partial or complete. This varies as per different aetiologies [19]. Lipodystrophy may be accompanied by metabolic disorders, including insulin resistance, diabetes mellitus, fatty liver and dyslipidaemia.

Other manifestations of metabolic derangements can include acanthosis nigricans due to SSIR, and eruptive xanthomas as a result of severe hypertriglyceridaemia and PCOS [20, 21]. PCOS secondary to SSIR states is not very 
uncommon. The prevalence of $1.5 \%$ of all PCOS phenotypes has been reported [22].

Severe insulin resistance contributes to PCOS-like phenotype due to both central and peripheral mechanisms. Compensatory hyperinsulinaemia acts directly on pituitary and affects $\mathrm{LH}$ pulsatility. Higher LH production leads to excess androgen production from theca cells (CYP45017 $\alpha$ activity). Excess androgens are produced by non-LH-mediated mechanisms as well. High insulin levels directly act on liver to reduce SHBG secretion, thus increasing proportion of free active androgens. FSH response of granulosa cells is also altered. All these metabolic changes lead to antral follicular arrest and PCOM.

Suspected cases should be referred to endocrinologist. Suspicion must arise when a fasting insulin level is more than $20.9 \mu \mathrm{g} / \mathrm{mL}$, and a peak insulin value after glucose load is above $209 \mu \mathrm{g} / \mathrm{mL}$ in a non-diabetic women with BMI less than $30 \mathrm{~kg} / \mathrm{m}^{2}$; likewise, suspicion must arise when insulin requirement is more than 3 units $/ \mathrm{kg}$ per day in diabetic women with $\mathrm{BMI}<30$ [17-21].

Diet control as well as insulin sensitizers, like thiazolidinediones specially pioglitazone [23-25], can be more helpful than metformin. However, therapeutic effect will be achieved only when adequate adipose depots are present. Low levels of serum leptin in patients with lipodystrophy and recombinant human leptin therapy may be highly effective in improving all metabolic derangements.

\section{Androgen-Secreting Tumours}

Classic PCOS is generally insidious in onset and presents with milder hirsutism, without much virilizing effects. If presentation is rather acute, with the rapid development of severe hyperandrogenism symptoms, virilization and menstrual irregularity, one must suspect an androgen-secreting tumour. Usually, the serum testosterone levels are more than $200 \mathrm{ng} / \mathrm{ml}$. This condition will be certainly a differential diagnosis of PCOS. Sertoli Leydig cell tumours are known to cause this picture; however, any other ovarian tumour may also present with signs of hyperandrogenism.

Androgen-secreting tumours are less commonly found arising from adrenal gland. Patients usually present with features of Cushing's syndrome as well as virilization.

This condition is also completely curable, and surgical treatment resolves all the symptoms.

\section{Drug-Induced PCOS}

Epilepsy in young girls is more susceptible to lead to PCOS when treated with valproic acid. This antiepileptic drug activates the transcription of $\mathrm{P} 450 \mathrm{c} 17$ and other enzymes involved in steroidogenesis [26, 27]. One meta-analysis suggested that in women treated with valproic acid for epilepsy, the incidence of PCOS is 1.95 -fold higher than in women treated with other drugs [28]. Valproic acid leads to weight gain; hence, this could be contributory factor in the development of PCOS phenotype. Most logically, the PCOS-like picture will regress after withdrawal of these drugs.

In addition to valproic acid effects, intrinsic neuroendocrine alterations in different types of epilepsy also may be responsible for PCOS phenotype.

Some androgenic drug therapy is associated with hirsutism and menstrual irregularities mimicking PCOS.

The exact cause of epilepsy is not well understood; hence, PCOS associated with this may not be completely curable.

\section{Conclusion}

Classic PCOS can be only controlled, and symptoms can be reduced. Many of them have recurrence of symptoms on cessation of therapy, and many others go on to develop comorbidities and health risks. Secondary causes of PCOS, even though rare, are completely curable. In clinical practice, it is worth searching for all these causes with appropriate investigations to rule out or confirm the same. If they are detected, then it will certainly be rewarding when patient gets completely cured of the undesirable symptoms. In complicated secondary cause, referral to concerned specialists will be more appropriate.

This account of various causes of secondary PCOS gives the broader view of PCOS phenotypes and gives an insight into pathophysiological aspects of PCOS [29]. The clinical categorization by HA-PODS nomenclature of both primary and secondary PCOS can minimize diagnostic and therapeutic pitfalls and will serve as a checklist to ensure that appropriate investigation is ordered and specific treatment is initiated as per diagnostic code. This will go a long way in establishing uniformity in diagnosis, treatment and research, which is the need of the hour. I hope and expect that further research may unfold some more unrevealed aspects of this condition towards better understanding of PCOS.

\section{References}

1. Stein I, Leventhal M. Amenorrhea associated with bilateral polycystic ovaries. Am J Obstet Gynecol. 1935;29:181-5.

2. National Institutes of Health. Evidence-based methodology workshop on polycystic ovary syndrome 2012. http://preventionnih. gov/workshops/2012/pcos/docs/PCOS_Final_Statement.pdf. Accessed 31 Jan 2013.

3. Rotterdam ESHRE/ASRM-Sponsored PCOS Consensus Workshop Group. Revised 2003 consensus on diagnostic criteria and long-term health risks related to polycystic ovary syndrome (PCOS). Hum Reprod. 2004;19:41-7. 
4. Azziz R. Controversy in clinical endocrinology: diagnosis of polycystic ovarian syndrome - the Rotterdam criteria are premature. J Clin Endocrinol Metab. 2006;91:781-5.

5. Azziz R, Carmina E, Dewailly D, et al. The Androgen Excess and PCOS Society criteria for the polycystic ovary syndrome: the complete task force report. Fertil Steril. 2009;91:456-88.

6. Khadilkar SS. Polycystic ovarian syndrome: is it time to rename pcos to HA-PODS? J Obstet Gynecol India. 2016;66(2):81-7.

7. Pasquali R, Gambineri A, Cavazza C, et al. Heterogeneity in the responsiveness to long-term lifestyle intervention and predictability in obese women with polycystic ovary syndrome. Eur J Endocrinol. 2011;164:53-60.

8. Moghetti P, Castello R, Negri C, et al. Metformin effects on clinical features, endocrine and metabolic profiles, and insulin sensitivity in polycystic ovary syndrome: a randomized, double-blind, placebo controlled 6 month trial, followed by open, long-term clinical evaluation. J Clin Endocrinol Metab. 2000;85:139-46.

9. Escobar-Morreale HF, Botella-Carretero JI, Alvarez-Blasco F, et al. The polycystic ovary syndrome associated with morbid obesity may resolve after weight loss induced by bariatric surgery. $\mathrm{J}$ Clin Endocrinol Metab. 2005;90:6364-9.

10. Skubleny D, Switzer NJ, Gill RS, et al. The impact of bariatric surgery on polycystic ovary syndrome: a systematic review and meta-analysis. Obes Surg. 2016;26:169-76.

11. Kosova G, Urbanek M. Genetics of the polycystic ovary syndrome. Mol Cell Endocrinol. 2013;373:29-38.

12. Choi YM, Kim WG, Kim TY, et al. Low levels of serum vitamin D3 are associated with autoimmune thyroid disease in pre-menopausal women. Thyroid. 2014;24:655-61.

13. Singla R, Gupta Y, Khemani M, et al. Thyroid disorders and polycystic ovary syndrome: an emerging relationship. Indian J Endocrinol Metab. 2015;19(1):25-9. https://doi.org/10.4103/22308210.146860 .

14. Hagag P, Hertzianu I, Ben-Shlomo A, et al. Androgen suppression and clinical improvement with dopamine agonists in hyperandrogenic-hyperprolactinemic women. J Reprod Med. 2001;46(7):678-84.

15. Azziz R, Sanchez LA, Knochenhauer ES, et al. Androgen excess in women: experience with over 1000 consecutive patients. J Clin Endocrinol Metab. 2004;89:453-62.

16. Cussons AJ, Stuckey BG, Walsh JP, et al. Polycystic ovarian syndrome: marked differences between endocrinologists and gynaecologists in diagnosis and management. Clin Endocrinol. 2005;62(3):289-95.

17. Semple RK, Savage DB, Cochran EK, et al. Genetic syndromes of severe insulin resistance. Endocr Rev. 2011;32:498-514.

18. Chan JL, Oral EA. Clinical classification and treatment of congenital and acquired lipodystrophy. Endocr Pract. 2010;16:310-23.

19. Garg A. Lipodystrophies: genetic and acquired body fat disorders. J Clin Endocrinol Metab. 2011;96:3313-25.

20. Handelsman Y, Oral EA, Bloomgarden ZT, et al. American Association of Clinical Endocrinologists. The clinical approach to the detection of lipodystrophy - an AACE consensus statement. Endocr Pract. 2013;19:107-16.

21. Semple RK. EJE PRIZE 2015: How does insulin resistance arise, and how does it cause disease? Human genetic lessons. Eur J Endocrinol. 2016;174:R209-23.

22. Pasquali R, Diamanti-Kandarakis E, Gambineri A. Management of endocrine disease: secondary polycystic ovary syndrome: theoretical and practical aspects. Eur J Endocrinol. 2016;175(4):R157-69.

23. Weedon MN, Ellard S, Prindle MJ, Caswell R, Allen HL, Oram R, Godbole K, Yajnik CS, Sbraccia P, Novelli G, Turnpenny P. An in-frame deletion at the polymerase active site of POLD1 causes a multisystem disorder with lipodystrophy. Nat Genet 2013;45(8):947-50

24. Semple RK, Savage DB, Cochran EK, et al. Genetic syndromes of severe insulin resistance. Endocr Rev. 2011;32:498-514.

25. Gambineri A, Semple RK, Forlani G, et al. Monogenic polycystic ovary syndrome due to a mutation in the lamin A/C gene is sensitive to thiazolidinediones but not to metformin. Eur J Endocrinol. 2008; 159:347-53.

26. Nelson-DeGrave VL, Wickenheisser JK, Cockrell JE, et al. Valproate potentiates androgen biosynthesis in human ovarian theca cells. Endocrinology. 2004;145:799-808.

27. Herzog AG. Menstrual disorders in women with epilepsy. Neurology. 2006;66:S23-8.

28. Hu X, Wang J, Dong W, et al. A meta-analysis of polycystic ovary syndrome in women taking valproate for epilepsy. Epilepsy Res. 2011;97(1-2):73-82.

29. Rosenfield RL, Ehrmann DA. The pathogenesis of polycystic ovary syndrome (PCOS): the hypothesis of PCOS as functional ovarian hyperandrogenism revisited. Endocr Rev. 2016;37(5):467-520.

Publisher's Note Springer Nature remains neutral with regard to jurisdictional claims in published maps and institutional affiliations.

\section{About the Author}

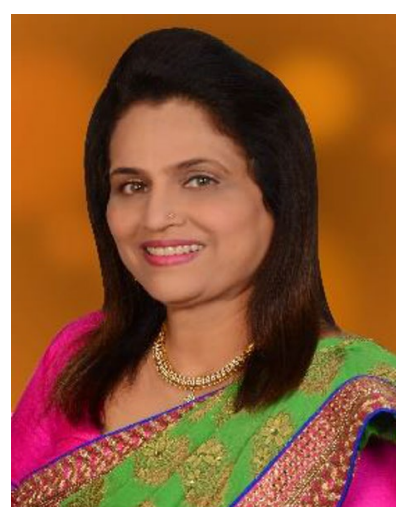

Dr. Suvarna Satish Khadilkar is the editor in chief of this journal. She is currently Professor and Head of the Department of Obstetrics and Gynecology and Consultant Gyne-Endocrinologist, Bombay Hospital Institute of Medical Sciences and Medical Research Centre, Mumbai. In the past, she worked as an Associate Professor and Unit Chief at JJ Group of Hospitals and Grant Medical College [GMC], Mumbai, and thereafter in the capacity of the Professor and Head in the Department of Obstetrics and Gynecology, Government Medical College, Kolhapur, Maharashtra. She has over 30 years of teaching experience as an undergraduate and postgraduate teacher and examiner, Mumbai University and Maharashtra University of Health Sciences. Pursuing her interest in endocrinology, she acquired Diploma in Endocrinology from the prestigious University of South Wales, UK, and has been appointed as a recognized teacher in endocrinology in University of South Wales. She is currently the treasurer of FOGSI, treasurer of Mumbai Ob-Gyn Society, and treasurer of Association of Medical Women in India [AMWI]. She has held many prestigious positions like President of Indian Menopause Society, Chairperson of Reproductive Endocrinology Committee of FOGSI, President of AMWI, Mumbai. She has published six textbooks and more than 70 articles at national and international levels. She is recipient of 30 awards for her research work including the Young Scientist Award. 